\title{
FLUORESCENCE EXCITATION OF ISOLATED, JET-COOLED 3-HYDROXYFLAVONE: THE RATE OF EXCITED STATE INTRAMOLECULAR PROTON TRANSFER FROM HOMOGENEOUS LINEWIDTHS
}

\author{
Niko P. ERNSTING and Bernhard DICK \\ Max-Planck-Institut für biophysikalische Chemie, Abteilung Laserphysik, D-3400 Göttingen, FRG
}

Received 18 January 1989

\begin{abstract}
The laser-induced fluorescence excitation of 3-hydroxyflavone, cooled by seeding in a supersonic expansion of He, has been investigated in the wavenumber range $28000-28500 \mathrm{~cm}^{-1}$. The vibronic origin of the $S_{0}-S_{1}\left(\pi \pi^{*}\right.$ ) electronic transition of the unperturbed normal molecular form was found at $28080.3 \mathrm{~cm}^{-1}(3560.21 \AA)$. The excitation spectrum is characterised by several progressions with vibrational spacing of $45 \mathrm{~cm}^{-1}$, and by severe spectral congestion at excess vibrational energy above $225 \mathrm{~cm}^{-1}$. The prominent vibronic bands are best represented by Lorentzian lineshapes. They are homogeneously broadened with widths (deconvoluted fwhm) varying from 4.1 to $2.6 \mathrm{~cm}^{-1}$. The corresponding vibronic lifetimes, in the range $1.3-2.0 \mathrm{ps,}$ are short because of excited-state intramolecular proton transfer.
\end{abstract}

\section{Introduction}

There has now been more than a decade of intensive and sometimes surprising research on the excited-state intramolecular proton transfer (ESIPT) reaction of 3-hydroxyflavone (3HF; cf. fig. 1). The attraction lay in the hypothesis that coplanarity of the phenyl ring with the main chromophore would speed up the reaction; therefore, a viscous barrier to tor-

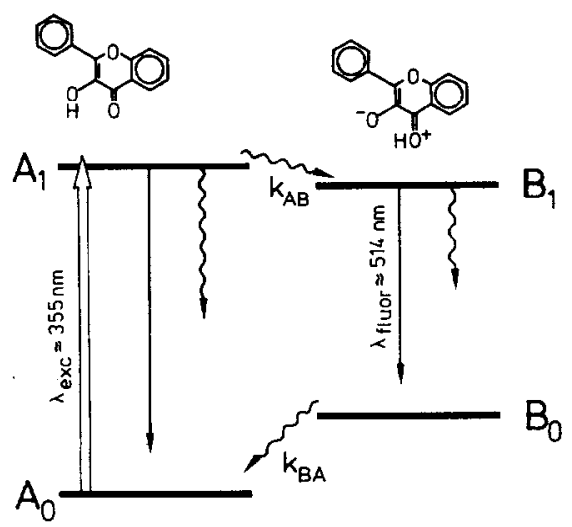

Fig. 1. Schematic energy level diagram for ESIPT in 3hydroxyflavone. sional phenyl relaxation towards coplanarity should result in normal, blue-violet fluorescence prior to intramolecular proton transfer followed by tautomer, yellow-green fluorescence [1]. However, the kinetics of the two fluorescence bands normally observed in hydrocarbon solvents required either two protontransfer channels, or else two conformations of 3-hydroxyflavone: one with an intramolecular hydrogen bond and the other with intermolecular hydrogen bonds (for the pertinent literature see refs. [2,3]). Also, pressure studies of tautomer emission yield in a hydrocarbon solvent did not reveal any effect of viscosity [4].

These inconsistencies were resolved by McMorrow and Kasha [5]. They showed that trace amounts of hydrogen-bonding solvent impurities lead to the presence of a series of ground-state solvates of $3 \mathrm{HF}$ with distinct spectral and dynamic properties. This sensitivity of 3HF to hydrogen-bonding perturbation may be a consequence of the geometrical structure for the intramolecular H-bond (five-membered ring) as well as of the electronic character of $3 \mathrm{HF}$ in its $S_{1}\left(\pi \pi^{*}\right)$ state (contribution of a pyrylium VB structure ). In extremely dry hydrocarbon solvents only the tautomer fluorescence was observed even at low temperatures. Hence the intrinsic proton transfer reac- 
tion occurs very fast. In an Ar matrix at $15 \mathrm{~K}$, the risetime of tautomer fluorescence could not be resolved with a temporal resolution of 2 ps [6].

More recent studies of ESIPT in 3HF follow two approaches:

(1) Spectroscopy in Ar matrices gives mainly energetic information on hydroxyflavones, and it characterises spectra and ESPT dynamics of specific $\mathrm{H}$ bonding solvate complexes $[2,3,7]$.

(2) Structural variation of the phenyl substituent $[3,8,9]$ again addresses the original question whether phenyl conjugation modulates the ESIPT transfer rate. However, in 3-hydroxychromone, which does not possess the phenyl substituent, the ESIPT reaction is still extremely fast [7]. Therefore time-resolved methods are also unlikely to distinguish the reaction rates of 3-hydroxyflavone derivatives with twisted phenyl groups. Other methods are needed to determine the rate of excited-state intramolecular proton transfer in these compounds and in $3 \mathrm{HF}$.

Electronic lifetimes $\leqslant 1$ ps should result in spectral vibronic bands with widths ( $\mathrm{fwhm}$ ) $\geqslant 5.3 \mathrm{~cm}^{-1}$. Resolved vibronic bands in the UV absorption spectrum of 3HF have not been observed so far, neither in Ar matrices [2] nor in Shpol'skii matrices [10]. This may be due to spectral congestion or to inhomogeneous broadening. In order to remove the latter, we examine isolated $3 \mathrm{HF}$ cooled in a supersonic jet using fluorescence excitation spectroscopy.

We determine the electronic origin of the $S_{0}-S_{1}$ $\left(\pi \pi^{*}\right)$ transition. Then we speculate briefly whether an active vibrational mode of $45 \mathrm{~cm}^{-1}$ in the $S_{1}$ state of $3 \mathrm{HF}$ may be assigned to phenyl torsion. Finally it is shown that the observed vibronic bands are well represented by Lorentzian lineshapes and are homogeneously broadened. From this, vibronic state reaction times of 1.3 to $2.0 \mathrm{ps}$ for the ESIPT reaction are inferred.

\section{Experimental}

3-hydroxyflavone (Eastman) was purified by repeated resublimation under high vacuum as described in ref. [2]. All subsequent handling of the solid, purified $3 \mathrm{HF}$ took place in a dry glove box under dried $\mathrm{N}_{2}$. After sublimation, the sample was transferred into the removable sample holder of the supersonic jet assembly. The sealed sample holder was then connected to the He reservoir of the jet assembly (see below). Finally, its sample end was opened while flushing with dry $\mathrm{He}$, and the sample holder was inserted into the preheated nozzle chamber.

The pulsed supersonic jet was actuated by a lowinductance solenoid. Sealing was accomplished by a Viton O-ring of $0.5 \mathrm{~mm}$ nominal internal diameter. The nozzle consisted of a small conical skimmer, with circular opening of $0.4 \mathrm{~mm}$ diameter and full cone angle of $90^{\circ}$, protruding into the O-ring. The skimmer had been punched out of Ta-foil $0.1 \mathrm{~mm}$ thick \#! This arrangement combines the advantages of $O$-ring sealing (high reproducibility and long lifetime) with those of "thin" nozzles (low dead volume and high aspect ratio; hence high pressure gradients). It has produced the coldest seeded supersonic expansions in this laboratory.

The nozzle assembly, including the sample in its holder, was heated to $400-420 \mathrm{~K}$. The resulting $3 \mathrm{HF}$ vapour pressures are estimated below 1.0 Torr from separate absorption measurements. He (4.8, dried by passing through a trap maintained at $77 \mathrm{~K}$ ) was used for the supersonic expansion at stagnation pressures of 1900-2600 Torr.

Fluorescence was excited $25 \mathrm{~mm}$ downstream from the nozzle by using a tunable pulsed dye laser (Lambda Physik FL2001). Pulse energy was $\approx 100$ $\mu \mathrm{J}$. The spectral intensity distribution of the laser output was measured with a high-resolution monochromator. The measured spectral profile is best represented by a Gauss curve with fwhm of $0.75 \mathrm{~cm}^{-1}$. For a spectral scan, the stepwidth of the laser was $0.0525 \AA$ corresponding to $0.41 \mathrm{~cm}^{-1}$. Laser wavelengths were calibrated using the monochromator which was in turn referred to a $\mathrm{Fe} / \mathrm{Ne}$ hollow cathode lamp. Absolute wavelengths for fluorescence excitation (table 1) are accurate to $0.05 \AA$.

Fluorescence was collected at right angles to the jet and laser beam by an aspheric quartz lens in combination with a spherical mirror. The tautomer fluorescence was mainly selected by a broad-band interference filter centered at $500 \mathrm{~nm}$ with a full bandpass of $100 \mathrm{~nm}$. Monitoring exclusively the "normal" fluorescence region $380-420 \mathrm{~nm}$, no emission above noise

\#1 We are grateful to Professor U. Even, Tel Aviv University, for this suggestion. 
Table 1

Wavenumber, bandwidth, and progressional assignment of vibronic bands in the fluorescence excitation spectrum of jet-cooled 3hydroxyflavone

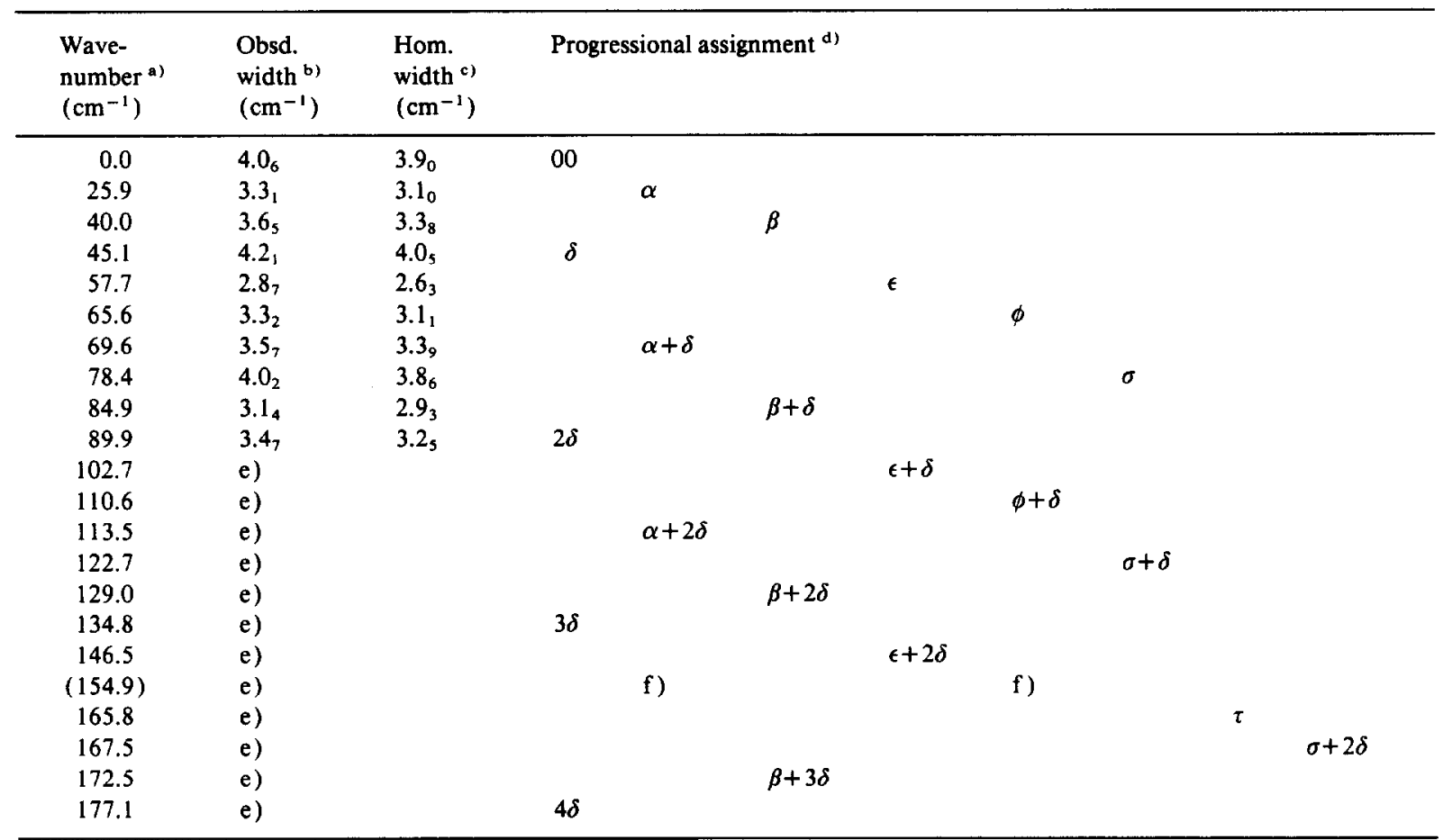

a) Relative to origin at $28080.3 \mathrm{~cm}^{-1}$ (in vacuo).

b) fwhm of Lorentzian lineshape from a simultaneous fit of the first 10 observed bands.

c) fwhm of Lorentzian lineshape, after deconvolution with a Gaussian lineshape with $0.8 \mathrm{~cm}^{-1}$ fwhm. Here the bandwidth reported in the preceding column was interpreted as belonging to a Voigt profile [17].

d) Progressions are denoted by Greek letters.

e) Not determined. "Overlapping.

was detected. The noise level corresponds to less than $0.5 \%$ of the signal obtained when tautomer fluorescence was monitored. The filtered emission was detected by a cooled photomultiplier (EMI 9558QA, S20 response). A small fraction of the laser beam split off by a quartz plate was monitored with a PIN diode to provide a reference. Both signals were sampled with boxcar integrators, digitized, and their ratio calculated on a computer. For each wavelength setting 20 events were averaged.

The pulsed supersonic jet had a pulse duration at the excitation region of $\approx 1 \mathrm{~ms}$ (measured by an ionisation gauge). For the best-dried sample and nozzle chamber, well-resolved excitation bands corresponding to uncomplexed, cold 3HF (see below) could be observed over this entire time window. However, a broad, unstructured background to fluorescence excitation was always present even at excitation energies below the lowest prominent vibronic band. The background signal intensity depended on the $3 \mathrm{HF}$ partial pressure (in the limited range available) in the same manner as the band intensity. Under less dry conditions, the background was becoming dominant regardless of the spectral window for detection, and resolved excitation bands could only be observed at early pulse times ( $<200 \mu$ s). Finally, addition of $\approx 1$ Torr of water completely obscured all spectral structure of fluorescence excitation, but strong green fluorescence was still observed.

Argon matrices were prepared at $10 \mathrm{~K}$ as in ref. [6]. Fluorescence spectra and fluorescence excitation spectra were recorded with a Spex Fluorolog system. 
Excitation spectra were corrected for spectral changes of excitation quantum flux; fluorescence spectra were corrected for the spectral response of the overall detection system.

\section{Results and discussion}

The fluorescence excitation spectrum and the fluorescence quantum distribution (over wavenumbers) of 3HF isolated in solid $\mathrm{Ar}$ are shown in fig. 2 (cf. also ref. [3]). The spectral region investigated in our supersonic-jet study is indicated by the horizontal bar.

The fluorescence excitation spectrum of cold 3HF in a seeded supersonic expansion of $\mathrm{He}$ is shown in fig. 3. Starting at lower excitation energy, a first prominent vibronic band is found at $28080.3 \mathrm{~cm}^{-1}$ $(3560.21 \AA)$. Wavenumbers of other spectral features will be given relative to this band. A region of well-resolved bands extends to relative energy of 111 $\mathrm{cm}^{-1}$. At higher energies up to $225 \mathrm{~cm}^{-1}$, the excitation spectrum shows a broad and increasing background with recognizable spectral structure. Beyond, the remnant structure merges into a broad excitation envelope.

A background to fluorescence excitation, caused by $3 \mathrm{HF}$, is also observed at excitation energies well below the first vibronic band. Its noise sets a lower limit

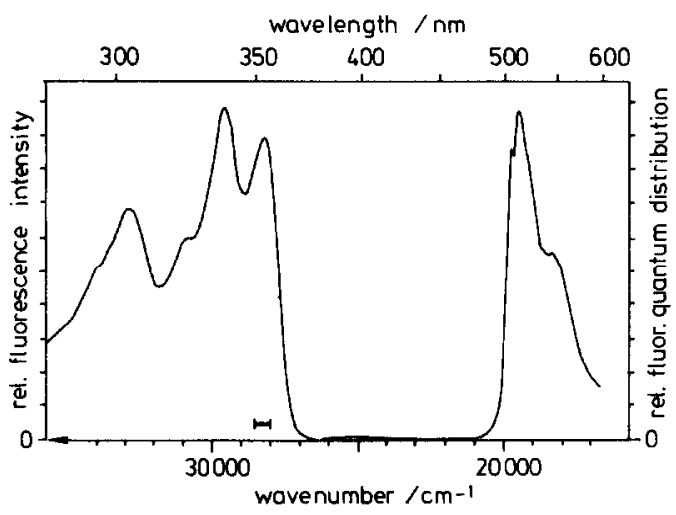

Fig. 2. Fluorescence spectrum (excited at $355 \mathrm{~nm}$ ) and fluorescence excitation spectrum (monitored at $514 \mathrm{~nm}$ ) of $3 \mathrm{HF}$ in an Ar matrix at $10 \mathrm{~K}$. The wavenumber region for excitation in the supersonic jet is indicated by a horizontal bar.

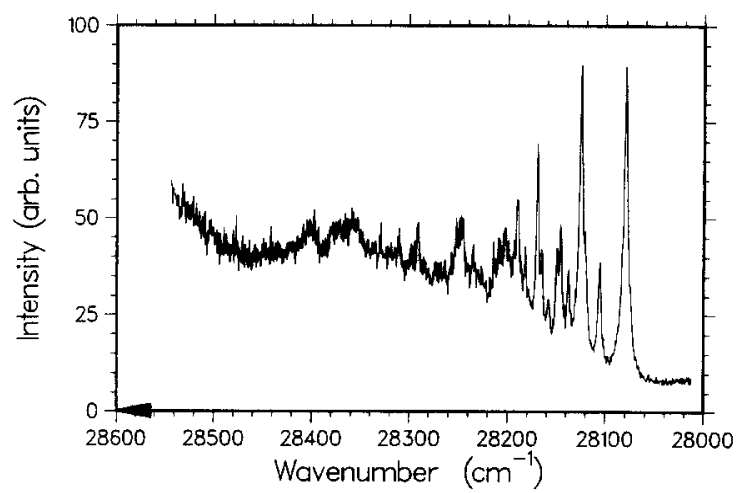

Fig. 3. Fluorescence excitation spectrum of $3 \mathrm{HF}$ in a pulsed supersonic expansion of $\mathrm{He}$ (stagnation pressure 2600 Torr).

to the detection signal for weak bands in an interval extending to $-100 \mathrm{~cm}^{-1}$. We attribute the unstructured background to the $3 \mathrm{HF} \cdot \mathrm{H}_{2} \mathrm{O}$ monosolvate complex [2] - present because of insufficiently dry handling conditions.

\subsection{Assignment to isolated $3 H F$}

Let us consider the tautomer fluorescence excitation spectra of hydroxyflavones in solid Ar [3, fig. 1A]. The spectra for 3-hydroxychromone and 4'-Me$3 \mathrm{HF}$ show a well-resolved relative maximum roughly $260 \mathrm{~cm}^{-1}$ below the spectral location of the first $S_{0^{-}}$ $\mathrm{S}_{1}$ absorption peak. The excitation spectrum of $3 \mathrm{HF}$ in solid Ar shows a shoulder at $358.5 \mathrm{~nm}$, also 260 $\mathrm{cm}^{-1}$ below the first absorption peak. It is reasonable to identify this relative spectral position with the electronic origin of the $S_{0}-S_{1}\left(\pi \pi^{*}\right)$ transition for the hydroxyflavones. Thus for $3 \mathrm{HF}$ in methylcyclohexane at $77 \mathrm{~K}$ [5], the origin should be located at 364.5 $\mathrm{nm}$, i.e. red-shifted by $470 \mathrm{~cm}^{-1}$ from its position in solid Ar. Increasing solvent polarizability for unpolar, non-hydrogen-bonding solvents lowers the $S_{0}-S_{1}$ transition energy [3]. The observed first vibronic band of isolated, jet-cooled $3 \mathrm{HF}$, at $356.0 \mathrm{~nm}$ fits well into this pattern; it is blue-shifted from the origin in solid $A r$ by $\approx 180 \mathrm{~cm}^{-1}$. Therefore we assign this band to the $0-0$ transition for the first $\pi \pi^{*}$ excitation of isolated $3 \mathrm{HF}$.

The absorption spectrum of $3 \mathrm{HF}$ vapour at $478 \mathrm{~K}$ [11] has a different shape compared to the fluorescence excitation spectrum in solid Ar. This is partly 
due to absorption from vibrational hot bands, leading to a loss of resolution. Therefore, the location of a first absorption inflection near $353 \mathrm{~nm}$ should still be consistent with an electronic origin at $356 \mathrm{~nm}$.

It is interesting to examine the red shift, of 180 $\mathrm{cm}^{-1}$, upon solvation of $3 \mathrm{HF}$ in solid Ar. This shift consists of a dispersive contribution (normally a red shift) and a contribution due to induced-dipole interaction [12]. The dispersive contribution of the $\mathrm{S}_{0}-$ $S_{1}$ transition in $3 \mathrm{HF}$ may be estimated from its oscillator strength $f$, the refractive index $n$ of solid $\mathrm{Ar}$ at $10 \mathrm{~K}$, and a cavity radius $a$. Using $f \approx 0.41$ and $n \approx 1.29$, the dispersive red shift should be $210 \mathrm{~cm}^{-1}$ for $a=4 \AA$ and only $110 \mathrm{~cm}^{-1}$ for a more reasonable $a=5 \AA$. Therefore, the additional contribution from induced-dipole interactions must be a (possibly small) red shift indicating a small increase of dipole moment upon electronic excitation. This is at variance with recent semiempirical calculations [13].

\subsection{Vibronic structure}

The spectral positions of vibronic bands in the excitation spectrum of jet-cooled 3HF are collected in table 1. A low-frequency vibrational mode in the excited state, of $45 \mathrm{~cm}^{-1}$, forms a dominant progression which is slightly blue-degraded and shows a small anharmonicity. It also builds progressions based on other low-frequency modes, e.g. of $25.9,57.7,65.6$ and $78.4 \mathrm{~cm}^{-1}$. No other low-frequency mode forms progressions in the resolvable part of the spectrum.

Similar progressions have been observed in excitation spectra of aromatic molecules having a phenyl torsion $[14,15]$. The change, upon electronic excitation, in the equilibrium dihedral angle between the phenyl ring and the main chromophore leads to nonzero Franck-Condon factors for vibronic transitions terminating in a series of torsional levels. For relatively small changes in the torsional equilibrium angle, a blue-degraded progression would result. Returning to $3 \mathrm{HF}$, it is tempting to associate the progression-forming $45 \mathrm{~cm}^{-1}$ mode with phenyl torsion. The ground-state dihedral angle in the crystal was determined to be $5.5^{\circ}[9]$. A change to coplanarity in the excited state would be consistent with our tentative assignment. However, the ground-state dihedral angle may be considerably larger in the isolated molecule (as in the case of biphenyl [16]).

\subsection{Lineshapes}

The spectral region with excess vibrational energy up to $90 \mathrm{~cm}^{-1}$ is shown in fig. 4 a. A simultaneous fit of the observed vibronic bands by Lorentzian lineshapes results in fig. $4 \mathrm{~b}$. The residuals of this Lorentzian fit are shown in fig. 4c, while the residuals (only) of a fit by Gaussian lineshapes are given below in fig. 4d. Comparing figs. $4 \mathrm{c}$ and $4 \mathrm{~d}$ it is apparent that Lorentzian lineshapes well describe the observed vibronic bands, while Gaussian lineshapes lead to systematic residuals in the band wings. The widths (fwhm) of the fitted Lorentzian lineshapes are also collected in table 1 ; they fall into the range 2.9-4.2 $\mathrm{cm}^{-1}$.

The shape of an observed band should be a convolution of the excitation laser lineshape, the rotational band envelope, and the homogeneous Loren-

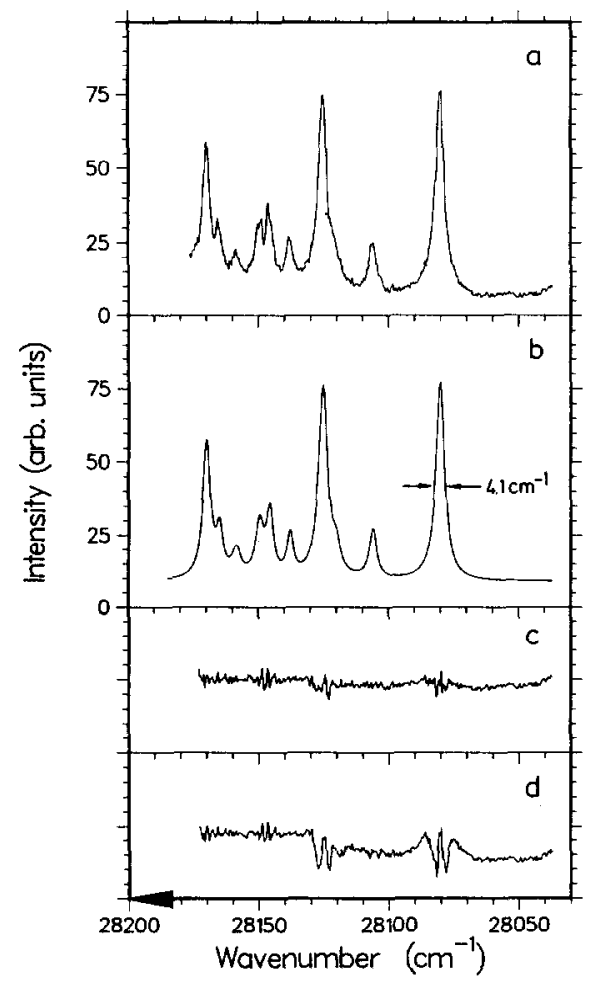

Fig. 4. (a) Fluorescence excitation bands of $3 \mathrm{HF}$ in a pulsed supersonic expansion of He. (b) The best fit of the observed spectrum by Lorentzian lineshape functions. (c) Residuals of the fit above. (d) Residuals of the best fit of the observed spectrum by Gaussian lineshape functions. 
tzian lineshape of the vibronic transition. For $3 \mathrm{HF}$ under the rotational cooling conditions of our experiment, we expect the rotational band envelope to be considerably narrower than the exciting laser line, which in turn is narrower than any of the observed bands. We conclude that the observed vibronic bands are homogeneously broadened. Deconvolution [17] with a Gaussian lineshape with fwhm of $0.8 \mathrm{~cm}^{-1}$, representing rotational band contour and exciting line, gives a homogeneous linewidth fwhm $_{\text {lum }}=3.9$ $\mathrm{cm}^{-1}$ for the electronic $\mathrm{S}_{0}-\mathrm{S}_{1}$ origin transition.

For an isolated molecule excited at a $0-0$ transition, the homogeneous linewidth is solely determined by the electronic lifetime $\tau$ : $\mathrm{fwhm}_{\text {hom }}=(2 \pi c \tau)^{-1}$. The electronic lifetime of isolated 3HF in its first excited state is limited by ESIPT; we obtain $\tau=1.4 \mathrm{ps}$ corresponding to a proton transfer rate constant (cf. fig. 1) of $k_{\mathrm{AB}}=0.74 \times 10^{12} \mathrm{~s}^{-1}$. This rate constant represents a close upper limit due to the assumptions about rotational band envelopes. On the other hand, time-resolved measurements of the tautomer fluorescence rise in solid Ar had established a certain lower limit $k_{\mathrm{AB}} \geqslant 0.5 \times 10^{12} \mathrm{~s}^{-1}[6]$.

\section{Conclusion}

We have shown that 3-hydroxyflavone cooled in a supersonic jet produces strong tautomer fluorescence following excitation in the electronic origin of the $\mathrm{S}_{0^{-}}$ $S_{1}$ transition at $28080.3 \mathrm{~cm}^{-1}$. The excitation spectrum of this fluorescence is characterised by several progressions in a low-frequency mode of $45 \mathrm{~cm}^{-1}$, starting at energies $\leqslant 80 \mathrm{~cm}^{-1}$ relative to the origin. The progression-forming vibrational mode of the excited state is tentatively assigned to phenyl torsion. The lowest ten vibronic bands are well fitted by Lorentzian lineshapes indicating that the bands are homogeneous. The measured width of the origin band,
$4.1 \mathrm{~cm}^{-1}$, corresponds to an upper limit for the ESIPT rate constant $k_{\mathrm{AB}}$ of $0.74 \times 10^{12} \mathrm{~s}^{-1}$.

\section{Acknowledgement}

We thank D. Ouw for expert technical assistance. We are indebted to Professor F.P. Schäfer for his constant support. The Deutsche Forschungsgemeinschaft is gratefully acknowledged for support through the Leibniz Prize Program.

\section{References}

[1] P.K. Sengupta and M. Kasha, Chem. Phys. Letters 68 (1979) 382.

[2] G.A. Brucker and D.F. Kelley, J. Phys. Chem. 91 (1987) 2856.

[3] G.A. Brucker and D.F. Kelley, J. Phys. Chem. 92 (1988) 3805.

[4] O.A. Salman and H.G. Drickamer, J. Chem. Phys. 75 (1981) 572 .

[5] D. McMorrow and M. Kasha, J. Phys. Chem. 88 (1984) 2235.

[6] B. Dick and N.P. Ernsting, J. Phys. Chem. 91 (1987) 4261.

[7] G.A. Brucker and D.F. Kelley, J. Phys. Chem. 91 (1987) 2862 .

[8] A.J.G. Strandjord, D.E. Smith and P.F. Barbara, J. Phys. Chem. 89 (1985) 2362.

[9] M.C. Etter, Z. Urbanczyk-Lipkowska, S. Baer and P.F. Barbara, J. Mol. Struct. 144 (1986) 155.

[10] D. McMorrow and M. Kasha, Proc. Natl. Acad. Sci. US 81 (1984) 3375.

[11] M. Itoh and H. Kurokawa, Chem. Phys. Letters 91 (1982) 487.

[12] E.G. McRae, J. Phys. Chem. 61 (1957) 562.

[13] D.A. Parthenopoulos and M. Kasha, Chem. Phys. Letters 146 (1988) 77

[14] J. Murakami, M. Ito and K. Kaya, J. Chem. Phys. 74 (1981) 6505.

[15] D.W. Werst, W.F. Londo, J.L. Smith and P.F. Barbara, Chem. Phys. Letters 118 (1985) 367.

[16] D. Kirin, J. Phys. Chem. 92 (1988) 3691.

[17] M.G. Zakaraya and J. Ulstrup, Opt. Commun. 68 (1988) 107. 